\title{
Cancer is an ancient disease: the case for better palaeoepidemiological and molecular studies
}

\section{Bishoy Faltas}

I read with great interest the recent Science and Society article (Cancer: an old disease, a new disease or something in between? Nature Rev. Cancer 10, 728-733 (2010)) $)^{1}$. In their conclusions, the authors interpreted the available evidence to suggest that malignancies were rare in antiquity because of a lack of exposure to carcinogens that are widespread in modern societies. I differ with the authors' interpretation of the available evidence and thus with their conclusions. The issue at hand can be examined by dissecting it into two distinct questions. The first: did cancer exist in antiquity? The answer is an emphatic yes; the authors mentioned more than 176 confirmed malignancies documented in the archaeological record ${ }^{1}$, providing ample evidence that cancer is an ancient natural phenomenon and is not solely a by-product of carcinogens that are abundant in today's industrial society. The next question: what was the prevalence of malignancies in ancient populations? Studying tumour palaeoepidemiology is uniquely challenging. A recent meta-analysis of all published palaeopathological studies of Egyptian mummies demonstrated how diagnostic uncertainty was a general problem in palaeopathological research and explained why clinical standards of diagnostic sensitivity and specificity were rarely applied ${ }^{2}$.
These methodological limitations make it difficult to reach the conclusions that are claimed by the authors regarding the frequency of cancer in antiquity. Recently, more carefully designed and methodologically sound palaeoepidemiological studies show that malignant tumours in the past were as prevalent as in modern populations. A recent study by Nerlich et al. examined the preserved skeletal remains of 905 individuals from two major ancient Egyptian necropolises spanning 3,200-500 вCE and also those of 2,547 individuals in ancient Germany dating back to $1400-1800 \mathrm{CE}$. This study established the presence of malignant tumours in spatially and temporarily different populations over the past 4,000 years with an age- and gender-adjusted frequency the same as that of a control group of the English population between 1900 and 1905 (REF. 3). Another study from the same group by Zink et al. examined the mummified remains of 325 adults in the ancient Egyptian necropolis of Thebes-west from 1,500 to $500 \mathrm{BCE}$ showed a higher prevalence than the same reference English population mentioned above ${ }^{4}$. We also have evidence from some studies that distinct types of malignant tumours such as multiple myeloma and nasopharyngeal carcinoma occurred at rates that are much higher than those in modern populations ${ }^{5}$.
In summary, cancer is an ancient disease and is not 'man-made'. There is strong evidence from palaeoepidemiological studies that cancer was at least as prevalent in ancient human populations as it is in our modern societies. More studies designed specifically to establish the epidemiology of cancer in ancient populations are needed to corroborate these findings. Such studies should apply the minimum standards that are proposed by Zweifel et al. ${ }^{2}$. Modern techniques such as molecular analysis can help to increase the specificity of diagnosis and shed light on the role of environmental factors in the pathogenesis of ancient cancers. For instance, if the Epstein-Barr virus (EBV) genome can be isolated from the cells of ancient nasopharyngeal carcinoma samples, it would confirm the aetiological role of EBV infection, similar to its established role in modern nasopharyngeal carcinoma ${ }^{6}$.

Bishoy Faltas is Clinical Senior Instructor, Department of Medicine, University of Rochester Medical Center, Rochester 14621, New York, USA. e-mail: bishoy.faltas@rochestergeneral.org doi: 10.1038/nrc2914-c1

1. David, A. R. \& Zimmerman, M. R. Cancer: an old disease, a new disease or something in between? Nature Rev. Cancer. 10, 728-733 (2010).

2. Zweifel, L., Buni, T. \& Ruhli, F. J. Evidence-based palaeopathology: meta-analysis of PubMed-listed scientific studies on ancient Egyptian mummies. Homo 60, 405-427 (2009).

3. Nerlich, A. G., Rohrbach, H., Bachmeier, B. \& Zink, A. Malignant tumors in two ancient populations: An approach to historical tumor epidemiology. Oncol. Rep. 16, 197-202 (2006).

4. Zink, A. et al. Malignant tumors in an ancient Egyptian population. Anticancer Res. 19, 4273-4277 (1999).

5. Capasso, L. L. Antiquity of cancer. Int. J. Cancer 113, 2-13 (2005).

6. Pathmanathan, R., Prasad, U., Sadler, R., Flynn, K. \& Raab-Traub, N. Clonal proliferations of cells infected with Epstein-Barr virus in preinvasive lesions related to nasopharyngeal carcinoma. N. Engl. J. Med. 333, 693-698 (1995).

Competing interests statement

The author declares no competing financial interests. 